\title{
Characteristics of electrostatic solitary waves observed in the plasma sheet boundary: Statistical analyses
}

\author{
H. Kojima ${ }^{1}$, Y. Omura ${ }^{1}$, H. Matsumoto ${ }^{1}$, K. Miyaguti ${ }^{1}$, and T. Mukai ${ }^{2}$ \\ ${ }^{1}$ Radio Atmospheric Science Center. Kyoto University, Kyoto. Japan \\ 2Institute of Space and Astronautical Science. Kanagawa, Japan
}

Received: 28 June 1999 - Revised: 21 November 1999 - Accepted: 22 November 1999

\begin{abstract}
We present the characteristics of the Electrostatic Solitary Waves (ESW) observed by the Geotail spacecraft in the plasma sheet boundary layer based on the statistical analyses. We also discuss the results referring to a model of ESW generation due to electron beams, which is proposed by computer simulations. In this generation model, the nonlinear evolution of Langmuir waves excited by electron bump-on-tail instabilities leads to formation of isolated electrostatic potential structures corresponding to "electron hole" in the phase space. The statistical analyses of the Geotail data, which we conducted under the assumption that polarity of ESW potentials is positive, show that most of ESW propagate in the same direction of electron beams, which are observed by the plasma instrument, simultaneously. Further, we also find that the ESW potential energy is much smaller than the background electron thermal energy and that the ESW potential widths are typically shorter than 60 times of local electron Debye length when we assume that the ESW potentials travel in the same velocity of electron beams. These results are very consistent with the ESW generation model that the nonlinear evolution of electron bump-on-tail instability leads to the formation of electron holes in the phase space.
\end{abstract}

\section{Introduction}

Electrostatic Solitary Waves (ESW) in the plasma sheet boundary layer were initially introduced as waveforms of Broadband Electrostatic Noise (BEN) observed by the Wave-Form Capture (WFC) receiver onboard the Geotail spacecraft (Matsumoto et al., 1994a; Kojima et al., 1994). They are series of impulsive and isolated pulses with the typical pulse width of a few milliseconds. These solitary waves correspond to the high frequency portion of the BEN spectra.

Solitary waves similar to the ESW observed by the
Geotail spacecraft have been also found by other spacecraft in various regions. The initial observation of the solitary waves was reported by Temerin et al. (1982). They found solitary structures in the electric field data observed by the S3-3 satellite in the polar magnetosphere at the altitude of $6030 \mathrm{~km}$. Koskinen et al. (1987) and Boström et al. (1988) conducted further detailed analyses of solitary waves observed at the same altitude in the polar region using Viking data. They showed that such solitary waves are associated with density depletion and that they correspond to negatively charged potentials. The discovery of the ESW by the Geotail spacecraft was followed by the observations of solitary waves in the polar region by the FAST, and POLAR spacecraft and in the solar wind/bow shock by the WIND spacecraft (Ergun et al., 1998; Franz et al., 1998; Tsurutani et al., 1998; Bale et al., 1998; Mangeney et al., 1999).

As we introduced in the above, solitary waves (solitary structures) are no longer special phenomena in space plasmas. The interesting point is the fact that similar solitary waves are observed in different regions with different plasma parameters. Therefore, what is the most important in the study of solitary waves is to establish the comprehensive generation model for explaining observations of all solitary waves in the different regions. Kojima et al. (1999a) pointed out that these solitary waves can be categorized into two groups. They are solitary waves corresponding positively charged potentials related to electron dynamics (Type I) and those corresponding negatively charged potentials related to ion dynamics (Type II). Solitary waves of the Geotail, POLAR, FAST spacecraft belong to the Type I and those of the S3-3 and Viking spacecraft belong to the Type II.

In order to identify the generation mechanism of the ESW observed by the Geotail spacecraft, computer simulations focusing on electron dynamics were conducted by Matsumoto et al. (1994a), and Omura et al. (1994). They demonstrated that generation of ESW is a result of nonlinear evolution of electron beam instabilities. 
They succeeded in reproducing the ESW waveforms and showed that such solitary waves are electron holes corresponding to positively charged potentials formed in the phase space at the final stage of electron beam instabilities. As the counterpart of these computer simulations for the ESW, ion holes in the phase space are also discussed in relation to the generation of solitary waves corresponding to negatively charged potentials observed by S3-3 and Viking spacecraft using computer simulations (Dupree, 1982; Hudson et al., 1983).

In order to confirm the generation mechanism of the ESW, it is necessary to discuss the detailed observation results comparing with the results of computer simulations. However, only a few event studies are not enough to discuss the natures of the ESW. In the present paper, we present the results of a comprehensive statistical analysis of ESW observed by the Geotail spacecraft in the plasma sheet boundary. In the following two sections, we summarize the results of Geotail observations and computer simulations focusing on ESW in the plasma sheet boundary. After that, we discuss the results of the statistical analyses referring to the results of computer simulations.

\section{Electrostatic Solitary Waves (ESW)}

The WFC receiver, which is one component of Plasma Wave Instrument (PWI) onboard the Geotail spacecraft has a capability for sampling waveforms with frequencies up to $4 \mathrm{kHz}$ (Matsumoto et al., 1994b). Waveform observations conducted by the WFC receiver revealed that the BEN in the Plasma Sheet Boundary Layer (PSBL) consists of a series of solitary pulses called "Electrostatic Solitary Waves (ESW)" (Matsumoto et al., 1994a; Kojima et al., 1994). Figure 1 shows that the representative waveforms of the ESW observed in the PSBL at (GSM$X$, GSM- $Y$, GSM- $Z)=\left(-12.5,0.5,0.7 R_{E}\right)$ on April 15 , 1995. Upper and lower panels show the parallel and perpendicular electric field components relative to the ambient magnetic field, respectively. The ESW shown in Figure 1 have coherent waveform structures in the parallel electric field component. Their pulse widths are almost equal to a few milliseconds. The perpendicular electric field component is quite weak compared with the parallel one. This waveform feature means that the ESW are the isolated potentials with one-dimensional structures traveling along the ambient magnetic field. These solitary waveform structures show the broadband nature in the frequency space, nonetheless, their contributions to the BEN spectra are only restricted to high frequency portions of the BEN spectra.

The properties of the observed ESW are summarized as follows (Kojima et al., 1997; Omura et al., 1999b).

1. Their typical pulse widths are a few milliseconds.

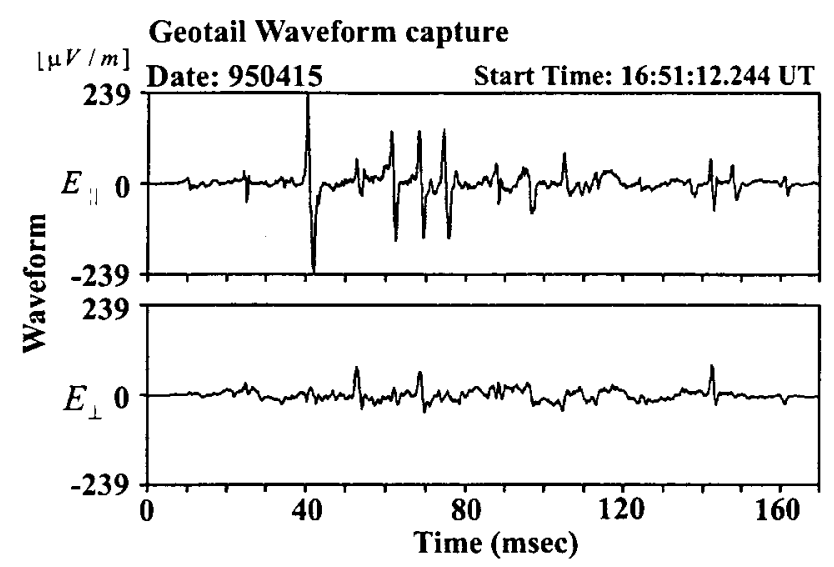

Fig. 1. Representative waveforms of ESW observed in the PSBL. Upper and lower panels show the waveforms of parallel and perpendicular components of electric fields relative to the ambient magnetic field, respectively.

2. They are purely electrostatic and any accompanying magnetic components are not observed.

3. They correspond to isolated electrostatic potentials traveling along the ambient magnetic field.

4. The potential structures of ESW are mostly onedimensional. However, occasionally, we also find ESW with a two-dimensional potential structure with large perpendicular electric field component.

5. The two-dimensional potential structures are more likely to be observed in the near tail region than in the distant tail region.

The solitary structures as shown in Figure 1 mean that these waves are highly nonlinear waves. Further, quick change of their wave properties such as their pulse widths and inter-pulse widths in the time scale of a few tens of milliseconds suggest that the ESW are closely related to electron dynamics rather than ion dynamics (Kojima et al., 1997).

\section{Generation model proposed by computer sim- ulations}

One-dimensional full particle computer simulations conducted by Matsumoto et al. (1994a) and Omura et al. (1994) succeeded in reproducing the waveforms of ESW. They demonstrated that the nonlinear evolution of electron two stream instability leads to the formation of isolated electrostatic potentials. The reproduced potentials are equivalent to the solution of the Bernstein-GreeneKruskal (BGK) mode (Bernstein et al., 1957). Omura et al. (1996) examined the nonlinear evolutions of several types of electron beam instabilities and showed that the bump-on-tail instability, which is more realistic as the 
model in the PSBL, also leads to the formation of the BGK mode.

The solution of the BGK mode is intrinsically onedimensional. However, Miyake et al. (1998) confirmed that the stable isolated potential structure can be also formed in two-dimensional space using the two-dimensional computer simulations. They found that the formed potentials have their uniform structure in the perpendicular direction relative to the ambient magnetic field. Miyake et al. (1998) concluded that the uniform perpendicular potential structures are formed through the diffusion process due to the electron cyclotron motion at the contact boundary of adjacent potential structures aligned in the perpendicular direction. Further, they also showed that the two-dimensional structure of the isolated potentials can be formed in the middle stage of the nonlinear evolution on the way to the formation of one-dimensional structure at the final stage.

The results from computer experiments on the ESW are summarized as follows (Omura et al., 1996; Miyake et al., 1998):

1. The nonlinear evolution of Langmuir waves excited by electron beam instabilities results in the formation of isolated positively charged potential structures, which are equivalent to the BGK solution corresponding to electron holes in the phase space.

2. Among several kinds of electron beam instabilities, the bump-on-tail instability is the most realistic and plausible generation mechanism of ESW in the PSBL region.

3. Before reaching the final stage of the nonlinear evolution, the two-dimensional potential structure can be seen in the simulation space. However, the diffusion process due to electron cyclotron motion force their two-dimensional structure to be reduced to the one-dimensional structure uniform in the perpendicular direction relative to the ambient magnetic field.

4. The generated isolated potentials travel along the ambient magnetic field in the same direction of electron beams with the same order of electron beam velocities.

The formation process of ESW in the two-dimensional space is schematically illustrated in Figure 2 based on the computer simulation results.

\section{Statistical analyses}

The waveforms reproduced by computer simulations agree well with observed waveforms of ESW. Further, Omura et al. (1999a) reported the several events in which the direction of observed electron beams corresponds to that of the ESW, demonstrating that computer simulations

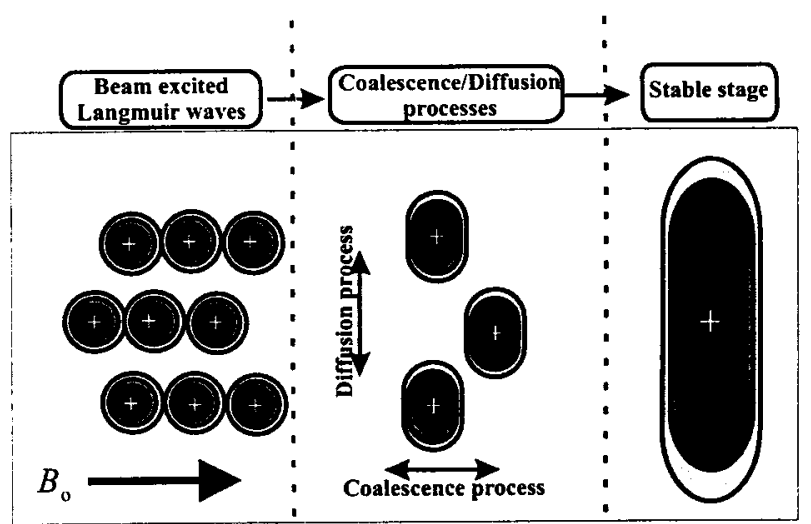

Fig. 2. Schematic illustration on the time evolution of ESW potential structures. In the nonlinear evolution of the beam excited Langmuir waves, the coalescence and diffusion processes take place in the direction parallel and perpendicular to the ambient magnetic field, respectively. These nonlinear processes result in the formation of isolated potential structures at the final stage.

explain the generation mechanism of ESW in the geomagnetic tail region very well. However, in order to conduct quantitative comparison between the results from computer simulations and the Geotail observations, it is necessary to collect more ESW and corresponding plasma data and to examine characteristics of ESW more comprehensively.

Since picking up ESW among huge amounts of Geotail waveform data needs tremendous efforts and time, we apply the pattern recognition method to select ESW waveforms from the Geotail data.

There exist many kinds of pattern recognition methods. However, since some of them require a lot of CPU resources, such methods are not usable for performing the statistical analyses of Geotail data, because of huge amounts of waveform data sets to be analyzed. In our statistical analyses, we make use of the method of a bit pattern matching. The brief description of this procedure is as follows (see Figure 3):

1. Making a bit pattern consisting of ' 0 ' and ' 1 ' for sample ESW waveform.

2. Making a bit pattern consisting of ' 0 ' and ' 1 ' for observed ESW waveforms.

3. Calculating Exclusive OR between the above two bit patterns.

4. Calculating the number of ' 1 ' from the result of the above Exclusive OR calculation (We call this number as "Distance").

When two patterns are completely the same, the Distance calculated at the fourth step of the procedure should be equal to zero. Thus, we can recognize the existence of ESW from small value of the Distance. The advantage of this method is not to use the complex 
erved

aveform

mple model waveform

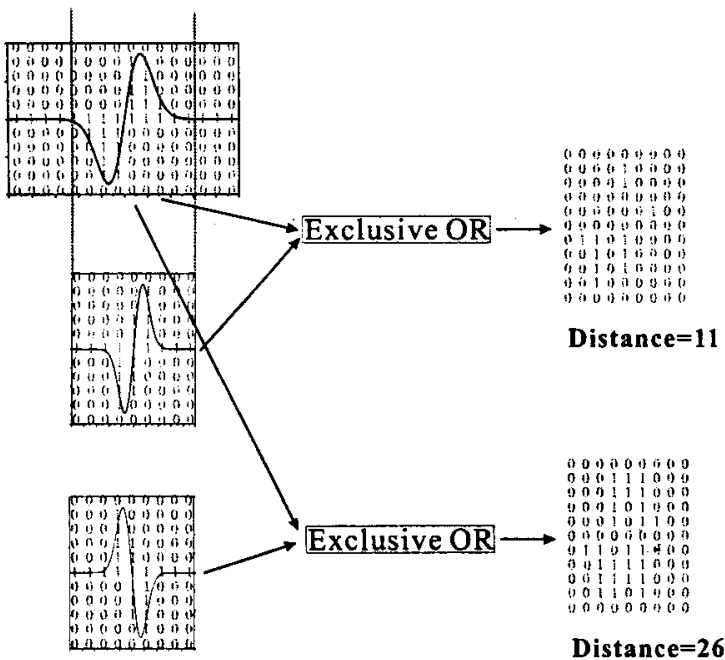

Fig. 3. Schematic illustration on the pattern matching method used in the present statistical analyses.

calculation such as Fourier transformation or Wavelet transformation, because what we have to do is to calculate Exclusive OR in each bit pattern. Therefore, this method does not need much CPU resource and it is very usable for the statistical analyses of ESW.

We apply this method to the waveform data of electric field parallel to the ambient magnetic field. As Kojima et al. (1999b) described, the propagation direction in the reference to the ambient magnetic field can be identified from the phase of ESW waveforms, if we assume the polarity of ESW potentials. Therefore, we prepare two different patterns with inverse phases each other as the sample pattern of ESW and calculate two distances by comparing these two sample patterns with the Geotail waveform" data (see Figure 3).

The sample model pattern for ESW is generated under the approximation of Gaussian potential model (Krasovsky et al., 1997). The Gaussian potential structure and corresponding spatial waveform of electric field are defined as

$$
\begin{aligned}
& \phi(z)=\phi_{\mathrm{o}} \exp \left(\frac{-z^{2}}{\lambda^{2}}\right) \\
& E(z)=-\operatorname{grad} \phi=\frac{2 \phi_{\mathrm{o}}}{\lambda^{2}} z \exp \left(\frac{-z^{2}}{\lambda^{2}}\right)
\end{aligned}
$$

Thus, the sample model waveform of ESW in the time domain is described as

$E(t)=\frac{2 \phi_{\mathrm{o}}}{\lambda} \frac{t}{\tau} \exp \left(\frac{-t^{2}}{\tau^{2}}\right)$

where $\tau=\frac{\lambda}{V}$, and $V$ is the velocity of ESW potentials. We generate the sample model pattern of ESW from Eq. (3) (see Figure 4).

The pulse width of ESW provides important information for evaluating potential scales and potential depths. The definition of the pulse width is ambiguous, but in

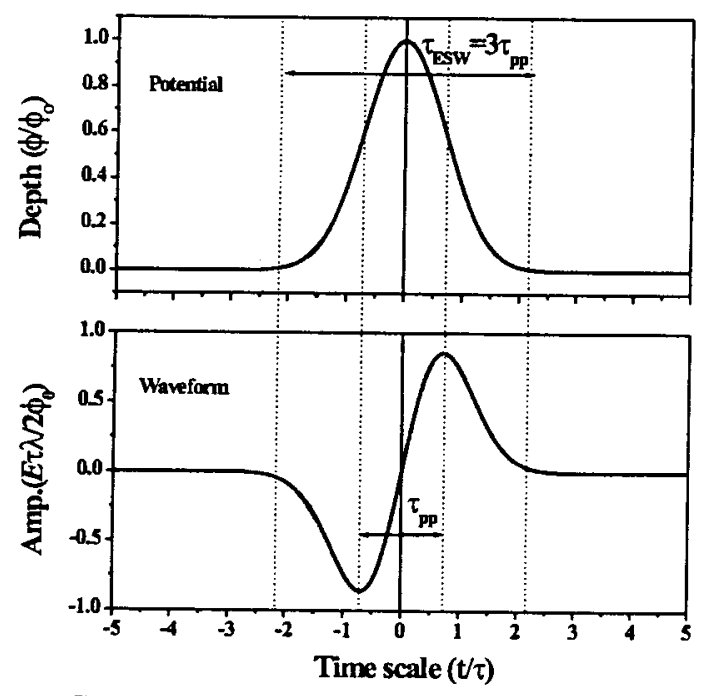

Fig. 4. Gaussian potential model and corresponding waveform. We define the ESW pulse width $\left(\tau_{\mathrm{ESW}}\right)$ as $\tau_{\mathrm{ESW}}=3 \tau_{\mathrm{pp}}$.

our statistical analyses, we make use of the time interval $\left(\tau_{\mathrm{pp}}\right)$ between two peak amplitudes $\left|E_{\| \max }\right|$ of ESW waveforms for its definition because they are easy to be identified from the data. $\left|E_{\| \max }\right|$ is obtained at $t= \pm \frac{\tau}{\sqrt{2}}$ in the above Gaussian potential model (see Figure 4$)$. Thus, we define the pulse width ( $\left.\tau_{\text {ESW }}\right)$ of ESW as three times of time interval of two peak amplitudes as follow

$\tau_{\mathrm{ESW}}=3 \times 2 \times \frac{\tau}{\sqrt{2}}=3 \times \tau_{\mathrm{pp}}$.

We applied the above pattern recognition method to the Geotail waveform data sets observed from September 1992 to December 1996 and picked up 275,808 ESW waveforms.

In the following sections, we compare the waveform data with electron velocity distributions detected by plasma detectors, simultaneously. While waveform data sampled with the frequency of $12 \mathrm{kHz}$ for one sampling period of 8.7 seconds can be obtained every 5 minutes, the time resolution of electron velocity distributions is 12 seconds. In order to avoid errors due to this difference of the time resolution, we only pick up the data sets with small value of the mean deviation in ESW amplitudes and their pulse widths observed for one sampling WFC period of 8.7 seconds. The mean deviation is defined as

$M D\left(x_{1}, \cdots, x_{N}\right)=\frac{1}{N} \sum_{j=1}^{N}\left|x_{j}-\bar{x}\right|$,

where $x_{1} \cdots, x_{N}$ and $\bar{x}$ denote peak amplitudes or pulse widths of ESW observed for one WFC sampling period of 8.7 seconds and their averaged values, respectively. In the following sections, we make use of only the data sets, which are satisfied with the condition that the ratio of the mean deviation value to the averaged value in one sampling. WFC period is less than $50 \%$. 


\section{Relation between electron beams and ESW}

In the generation model of ESW based on the results of computer simulations focusing on electron beam instabilities, the excited ESW potentials travel along the ambient magnetic field in the same direction of electron beams. We can examine this fact by comparing statistically the ESW traveling directions with the electron beam directions, which are observed by the Low Energy Particle experiment (LEP) onboard Geotail spacecraft. The LEP has the capability to measure threedimensional electron velocity distributions in the energy range from several $\mathrm{eV} / \mathrm{q}$ to $43 \mathrm{keV} / \mathrm{q}$ (Mukai et al., 1994).

The trapping effects due to the formed potentials result in formation of high energy tail of electron velocity distributions in the direction of electron beams (Omura et al., 1996). Omura et al. (1999a) introduced two events, which show that the ESW traveling direction is the same with the direction of high energy tail of the reduced electron velocity distributions by comparing ESW waveforms with LEP data. In order to confirm their results statistically, we performed the statistical analyses on the relation between ESW traveling directions and electron beam directions.

The Geotail PWI does not have the capability for identifying the polarity of ESW potentials and their traveling direction. However, when we assume a positive potential formed by electron holes based on the computer simulation results, we can identify the traveling direction of ESW using the electric field antenna polarity (Detailed procedure should be referred to Kojima et al. [1999b]).

In this analysis, we pick up the cases in which the detected electron beam velocities are beyond $10,000 \mathrm{~km} / \mathrm{s}$ in order to select the events in which we can clearly identify electron beam components.

From this statistical analysis, we found that $88 \%$ of observed ESW travel in the same direction of electron beams. This quite good correlation is very consistent with the ESW generation due to electron beams. This is the first statistical result for showing the good correlation between ESW observed in the PSBL and electron beam directions.

Note that if we assume the negatively charged ESW potentials instead of positively charged ones, the results become completely inverse. This means that $88 \%$ of ESW travel in the opposite direction to electron beams. This result is very strange, because it is very unlikely that excited potentials travel in the different direction to electron beams as their free energy source. Thus, we can conclude that our assumption on the polarity of ESW potentials is quite reasonable.

\section{Scales and depth of ESW potentials}

Based on the results of computer simulations, the electron bump-on-tail instability is the most realistic generation mechanism of the ESW in the PSBL. In the bump-on-tail instability, the potential energies of the generated isolated potentials are small relative to those of potentials generated due to the two-stream type instability.

The pattern recognition method introduced in section 4 allows us to estimate potential energy of the ESW by using the information of ESW amplitudes and potential widths.

We estimate electron beam velocities $\left(V_{\mathrm{eb}}\right)$ from LEP data in order to calculate potential width of ESW (Detailed procedure for estimating the electron beam velocity is formed in Omura et al. (1999a)). Since the Geotail PWI does not have the capability to identify traveling velocities of ESW, we assume that the ESW traveling velocity is the same with electron beam velocities based on the computer simulation results.

On the other hand, since $\left|E_{\max }\right|$ can be described as

$E_{\max }=E\left(\frac{\tau}{\sqrt{2}}\right)=\frac{1}{\lambda} \sqrt{\frac{2}{e}} \phi_{\mathrm{o}}$

by using Eq. (4), we can obtain the potential energy as

$\phi_{\mathrm{o}}=\frac{\sqrt{e}}{6}\left|E_{\max }\right| \tau_{\mathrm{ESW}} V_{\mathrm{eb}}$

Figure 5 is the scatter plot showing the distribution of ESW potential energy versus their potential widths obtained by the above Eqs. (4), and (7). The symbols of diamonds and crosses represent earthward and tailward traveling ESW, respectively. In Figure 5, it is clear to see that most of ESW potential energies are distributed in the range less than $4 \mathrm{eV}$. This value is very small relative to the background electron thermal energies. Since the typical thermal energies of background electrons in the PSBL is a few hundreds of eV, this result means that the ESW do not play any important roles in thermalization of electrons and ions in the PSBL. However, it agrees with the computer simulation result on the bump-on-tail instability, which shows that energies of ESW potential generated due to the bump-on-tail instability are very small relative to background electron thermal energies.

Figure 6 shows the relation between potential widths of the ESW and electron Debye length $\left(\lambda_{\mathrm{e}}\right)$. Since the parent waves of the ESW generated by the bump-on-tail instability are the Langmuir waves, the potential widths should be related to the electron Debye length. The relation of potential width and electron Debye length is roughly estimated as follows.

The relation between wavenumber $(k)$ of the excited Langmuir waves and electron beam velocity $\left(V_{\mathrm{eb}}\right)$ can be approximately written as 


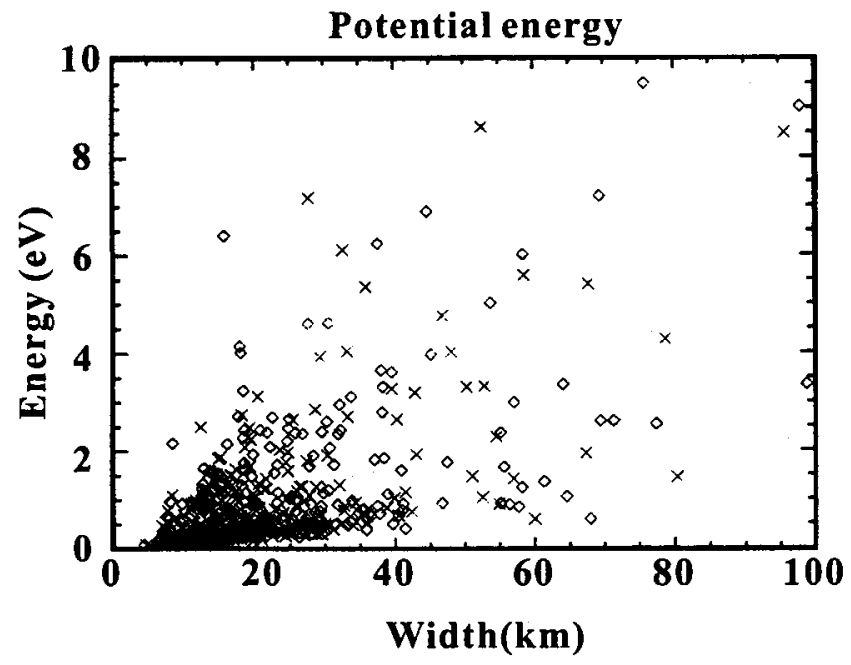

Fig. 5. Scatter plot on potential energies of ESW. Obtained potential energies are mostly confined in the energy range below $4 \mathrm{eV}$. This means that ESW potential energies are much smaller than background electron thermal energies and that ESW do not have an important role in the themalization of electrons in the PSBL.

$\frac{\Pi_{\mathrm{pe}}}{k} \sim V_{\mathrm{eb}}$

where $\Pi_{\mathrm{pe}}$ is the electron plasma frequency. Since $\Pi_{\mathrm{pe}}=$ $\frac{v_{\text {the }}}{\lambda_{\mathbf{e}}}$, we can obtain the relation of potential width $(w)$ and electron Debye length as

$\frac{w}{\lambda_{\mathrm{e}}} \sim 2 \pi \frac{V_{\mathrm{eb}}}{v_{\mathrm{the}}}$,

where $v_{\text {the }}$ is electron thermal velocity.

Since the typical ratio of $\frac{V_{\mathrm{b}}}{v_{\text {the }}} \sim 3$ in the typical PSBL (Omura et al. 1999a), we can estimate $\frac{w}{\lambda_{e}}$ as .

$\frac{w}{\lambda_{\mathrm{e}}} \sim 2 \pi \frac{V_{\mathrm{b}}}{v_{\text {the }}} \sim 20$.

Figure 6 shows that the above rough estimation agrees well with observation results. Most of observed data focus on the range of $w / \lambda_{\mathrm{e}}<60$. This agreement between the above rough estimation and observation results on the ratio of potential widths to Debye length strongly supports the generation model of the bump-ontail instability, which was proposed by computer simulations(Omura et al., 1996). This result also shows that our assumption that ESW potentials travel in the almost same speed with electron beams is reasonable.

In Figure 6, we also see some data with huge values of $w / \lambda_{\mathrm{e}}$ beyond 100. Sometimes we see such huge ESW in the PSBL. The relation between small ESW and huge ESW is still unclear.

\section{Conclusions and Discussion}

The present paper discussed the statistical features of ESW observed by the Geotail spacecraft in the plasma

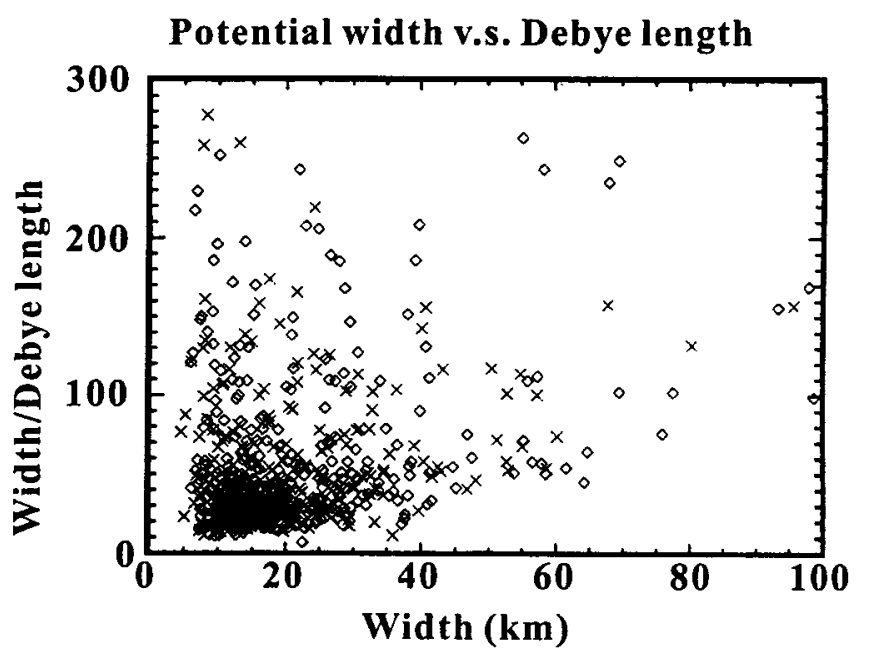

Fig. 6. Scatter plot on the ratio of ESW potential width to electron Debye length.

sheet boundary based on the statistical analyses. In order to realize the practical analysis of ESW, we applied the pattern recognition method conducted by the bit pattern matching to the Geotail waveform data.

The results of our statistical analyses are summarized as follows:

1. ESW traveling direction is almost the same with electron beam direction.

2. Potential energies of ESW are mostly less than $4 \mathrm{eV}$, which is much smaller than the background thermal energies.

3. The ratio of ESW potential length to the electron Debye length is mostly less than 60 . This ratio agrees with the rough theoretical estimation in the case of the bump-on-tail instability.

Omura et al. (1996) pointed out two plausible processes for the formation of ESW using computer simulations. They are the electron two-stream instability and the electron bump-on-tail instability. The above results are significantly consistent with the results of computer simulations focusing on the bump-on-tail instability. In fact, electron velocity distributions detected by LEP while observing ESW, do not cause the two-stream instability, because electron beam densities are very small relative to background electrons. Figure 7 shows the statistical results on the ratio of electron beam densities to background electron densities while we observe ESW in the PSBL. We find that electron beam densities are very small relative to background electron densities. The ratio is mostly less than $10 \%$. Further, as we discussed in Section 5, the typical electron beam velocities are almost three times higher than background electron thermal velocities in the PSBL. The above plasma environment is satisfied with the condition of the bump-ontail instability. 


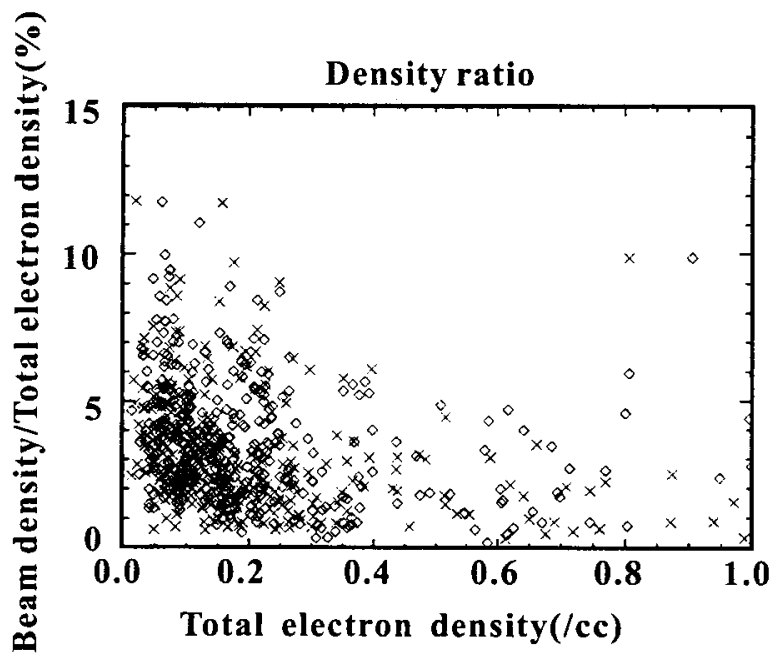

Fig. 7. Scatter plot on the ration of electron beam density to total electron density in the PSBL. The electron beam densities related to ESW are less than $10 \%$ of total electron densities.

By combining our results of the Geotail observations and computer simulations, we can conclude that the ESW in the PSBL are the results of nonlinear evolution of the electron bump-on-tail instability and that they are "electron holes" in the phase space, which correspond to positively charged isolated potentials.

The two-stream instability is the plausible generation mechanism for solitary waves observed by the FAST or POLAR spacecraft in the polar magnetosphere, because there exist energetic and dense electron beams in this region. In the two-stream instability, the potential energies of formed solitary waves are expected to be much larger than those in the bump-on-tail instability. This expectation is consistent with the observation results, because amplitudes of solitary waves observed by the FAST or POLAR are much larger than those of ESW observed by the Geotail in PSBL.

The waveform observations with high time resolutions by several spacecraft revealed that solitary wave structures are common phenomena in space plasmas. Their waveforms are very similar to each other. However, we also find slight different features among them such as corresponding potential polarities, pulse widths, and amplitudes. Our final goal is to establish comprehensive model for generating various kinds of solitary waves observed in various regions with various plasma parameters. Further close collaborations of spacecraft observations and computer simulations are needed in the future.

Acknowledgements. This paper was presented in the international workshop on Nonlinear Waves and Chaos in Space Plasmas, which was held at San Diego in March 1999. The authors thank the workshop conveners, B. T. Tsurutani and P. H. Diamond for organizing the workshop. We would like to thank the ISAS/NASA Geotail mission project team for their support. We appreciate S. Kokubun and late T. Yamamoto providing MGF data to identify ESW propagation direction. This research was supported by grant-in-aid 08404027.

\section{References}

Bale, S. D., P. J. Kellogg, D. E. Larson, R. P. Lin, K. Goetz, and R. P. Lepping, Bipolar electrostatic structures in the shock transition region: Evidence of electron phase space holes, Geophys. Res. Lett., 25, 2929-2932, 1998.

Bernstein, I. B., J. M. Greene, and M. D. Kruskal, exact nonlinear plasma oscillations, Phys. Rev., 108, 546-550, 1957.

Boström, R., G. Gustafsson, B. Holback, G. Holmgren, and H. Koskinen, and P. Kintner, Characteristics of solitary waves and weak double layers in the magnetospheri c plasma, Phys. Rev. Lett., 61, 82-85, 1988.

Dupree, T. H., Theory of phase-space density holes, Phys. Fluids, $25,277-289,1982$.

Ergun, R. E., C. W. Carlson, J. P. McFadden, F. S. Mozer, G. T. Delory, W. Peria, C. C. Chaston, M. Temerin, I. Roth, L. Muschietti, R. Elphic, R. Strangeway, R. Pfaff, C. A. Cattell, D. Klumpar, E. Shelley, W. Peterson, E. Moebius, and L. Kistler, FAST satellite observations of large-amplitude solitary structures, Geophys. Res. Lett., 25, 2041-2044, 1998.

Franz, J. R., P. M. Kintner, J. S. Pickett, POLAR observations of coherent electric field structures, Geophys. Res. Lett., 25, 1277-1280, 1998.

Hudson, M. K., W. Lotko, I. Roth, and E. Witt, Solitary waves and double layers on auroral field lines, J. Geophys. Res., 88, 916-926, 1983.

Kojima, H., H. Matsumoto, T. Miyatake, I. Nagano, A. Fujita, L. A. Frank, T. Mukai, W. R. Paterson, Y. Saito, S. Machida, and R. R. Anderson, Relation between electrostatic solitary waves and hot plasma flow in the plasma sheet boundary layer: GEOTAIL Observations, Geophys. Res. Lett., 21, 2919-2922, 1994.

Kojima, H., H. Matsumoto, S. Chikuba, S. Horiyama, M. AshourAbdalla, and R. R. Anderson, GEOTAIL Waveform Observations of Broadband/Narrowband Electrostatic Noise i $n$ the Distant Tail, J. Geophys. Res., 102, 14439-14455, 1997.

Kojima, H., H. Matsumoto, and Y. Omura, Electrostatic solitary waves observe $\mathrm{d}$ in the geomagnetic tail and other regions, $A C$ cepted for the publication in Adv. In Space Res., 1999a.

Kojima H., K. Ohtsuka, H. Matsumoto, Y. Omura, R. R. Anderson, Y. Saito, T. Mukai, S. Kokubun, and T. Yamamoto, Plasma waves in slow-mode shocks observed by Geotail spacecraft, Adv. In Space Res., 24, No. 1, 51-54, 1999b.

Koskinen, H. E. J., R. Boström, and B. Holback, Viking observations of solitary waves and weak double layers on auroral field lines, Phys. of Space Plasmas, SPI conference proceedings and. reprints series, $7,147-156,1987$.

Krasovsky, V. L., H. Matsumoto, and Y. Omura, BernsteinGreene-Kruskal analysis of electrostatic solitary waves observed by Geotail spacecraft, J. Geophys. Res., 102, 22131-22139, 1997.

Mangeney, A., C. Salem, C. Lacombe, J. -L. Bougeret, C. Perche, R. Manning, P. J. Kellog, K. Geotz, S. J. Monson, and J. M. Bosquend, WIND observations of coherent electrostatic waves in the solar wind, Annales Geophysicae, 17, 307-320, 1999.

Matsumoto, H., H. Kojima, T. Miyatake, Y. Omura, M. Okada, I. Nagano, and M. Tsutsui, Electrostatic Solitary Waves (ESW) in the Magnetotail : BEN Wave forms obs erved by GEOTAIL, Geophys. Res. Lett., 21, 2915-2918, 1994 a.

Matsumoto, H., I. Nagano, R. R. Anderson, H. Kojima, K. Hashimoto, M. Tsutsui, T. Okada, I. Kimura, Y. Omura, and M. Okada, Plasma Wave Observations with GEOTAIL Spacecraft, J. Geomag Geoelectr, 46, 59-95, 1994b.

Miyake, T., Y. Omura, H. Matsumoto, and H. Kojima, Twodimensional computer simulations of electrostatic solitary waves observed by Geotail spacecraft, J. Geophys. Res., 103, 11841-11850, 1998. 
Mukai, T., S. Machida, Y. Saito, M. Hirahara, T. Terasawa, N. Kaya, T. Obara, M. Ejiri, and A. Nishida, The low energy particle (LEP) experiment onboard the Geotail satellite, J. Geomag Geoelectr, 46, 669-692, 1994.

Omura, Y., H. Kojima, and H. Matsumoto, Computer simulation of Electrostatic Solitary Waves: A nonlinear model of broadband electrostatic noise, Geophys. Res. Lett., 21, 2923-2926, 1994.

Omura, Y., H. Matsumoto, T. Miyake, and H. Kojima, Electron beam instabiliti es as generation mechanism of electrostatic solitary waves in the magnetotai 1, J. Geophys. Res., 101, 26852697, 1996.

Omura, Y., H. Kojima, N. Miki, T. Mukai, H. Matsumoto, and R. R. Anderson, Electrostatic Solitary Waves Carried by Diffused Electron Beams Observed by GEOTAIL Spacecraft, $J$. Geophys. Res., 104, 14627-14637, 1999a.

Omura, Y., H. Kojima, N. Miki, and H. Matsumoto, Twodimensional electrostatic solitary waves observed by Geotail in the magnetotail, Adv. In Space Res., 24, No. 1, 55-58, 1999b.

Temerin, M., K. Cerny, W. Lotko, and F. S. Mozer, Observations of double layers and solitary waves in the auroral plasma, Phys. Rev. Lett., 48, 1175-1179, 1982.

Tsurutani, B. T., J. K. Arballo, G. S. Lakhina, C. M. Ho, B. Buti, J. S. Pickett, and D. A. Gurnett, Plasma waves in the dayside polar cap boundary layer: Bipolar and monopolar electric pulses and whistler mode waves, Geophys. Res. Lett., 25, 4117 . 4120, 1998. 